\title{
MAGNETIZATION RELAXATION IN A FOUR-LEVEL SYSTEM
}

\author{
A.M. WiTowski \\ Institute of Experimental Physics, Warsaw University \\ Hoża 69, 00-681 Warszawa, Poland
}

\begin{abstract}
The relaxation of magnetization in a four-level system (e.g. $\mathrm{Co}^{2+}$ in II-VI compounds) is discussed. It is shown that in the heat pulse experiment the slowest relaxation time is basically observed.

PACS numbers: $76.30 . F c$
\end{abstract}

The relaxation of magnetization in a system with $\mathrm{Mn}^{2+}$ has been recently investigated both theoretically [1] and experimentally in CdMnTe [2]. The studied materials are diluted magnetic semiconductors based on CdTe with $\mathrm{Mn}$ as a magnetic ion. In such a case the $\mathrm{Mn}^{2+}$ ground state is an orbital singlet with $S=5 / 2$ (e.g. [3]) leading in a magnetic field to six spin split levels in the ground state. In the experiment the sample was placed in the pick-up coil [2]. The thermal equilibrium between the spins and the lattice was disturbed with the nonresonant laser pulse (rise of the lattice temperature). It has been found that the time dependence of magnetization approaching its thermal equilibrium value is almost exponential. Generally, for an $n$-level system the relaxation is described as a linear combination of $n-1$ exponential functions of time leading to $n-1$ different relaxation rates [4]. Which rate will be the dominant one for a given relaxation mechanism depends on initial conditions. For $\mathrm{Mn}^{2+}$ six spin levels it is difficult to compare the numerical solution of rate equations [1] with expected five relaxation rates, therefore in the present work we choose a more simple four-level system, namely $\mathrm{Co}^{2+}$ in II-VI compounds (Fig. 1). A free $\mathrm{Co}^{2+}$ ion has $3 d^{7}$ configuration leading to a ${ }^{4} F$ ground state, which is split by the crystal field into an orbital singlet ${ }^{4} A_{2}$ and higher in energy lying orbital triplets [5].

For the sake of clarity we assume only magnetic relaxation [6], it means that only transitions shown in Fig. 1 are allowed. In such a case it is easy to calculate relaxation rates from the following rate equations:

$$
\begin{aligned}
& \frac{\mathrm{d} n_{1}}{\mathrm{~d} t}=-3 W_{\mathrm{u}} n_{1}+3 W_{\mathrm{d}} n_{2}, \quad \frac{\mathrm{d} n_{2}}{\mathrm{~d} t}=3 W_{\mathrm{u}} n_{1}-\left(3 W_{\mathrm{d}}+4 W_{\mathrm{u}}\right) n_{2}+4 W_{\mathrm{d}} n_{3}, \\
& \frac{\mathrm{d} n_{3}}{\mathrm{~d} t}=4 W_{\mathrm{u}} n_{2}-\left(4 W_{\mathrm{d}}+3 W_{\mathrm{u}}\right) n_{3}+3 W_{\mathrm{d}} n_{4}, \quad \frac{\mathrm{d} n_{4}}{\mathrm{~d} t}=3 W_{\mathrm{u}} n_{3}-3 W_{\mathrm{d}} n_{4},(1)
\end{aligned}
$$

where $W_{\mathrm{u}}$ and $W_{\mathrm{d}}$ denote functions describing probabilities of a transition up and down, respectively. The temporal behavior of level populations $n_{i}(t)$ is described by the sum

$$
n_{i}(t)=n_{i 0}+\sum_{j} a_{i j} \exp \left(-\lambda_{j} t\right) .
$$




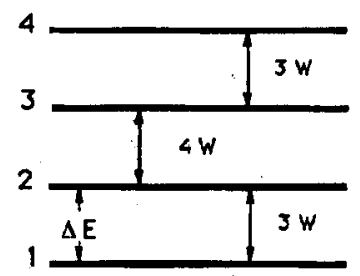

Fig. 1. The energy levels of the $\mathrm{Co}^{2+}$ ground state in magnetic field $B$ and possible transitions between the levels for the magnetic relaxation.

Relaxation rates $\lambda_{j}$ are solutions of the cubic equation coming from Eqs. (1). Assuming the direct relaxation process (emission (down) and absorption (up) of one phonon with an energy equal to the spin splitting energy $\Delta E=g \mu_{\mathrm{B}} B$ ) and the phonon density of states described by the Debye model, the transition probabilities can be written in the form (e.g. [4]):

$$
\begin{aligned}
& W_{\mathrm{d}}=C(\Delta E)^{3}\{1+1 /[\exp (\Delta E / k T)-1]\}, \\
& W_{\mathbf{u}}=C(\Delta E)^{3} /[\exp (\Delta E / k T)-1],
\end{aligned}
$$

where $C$ is treated as a parameter (in our calculations taken as 1) proportional to the matrix element of electronic wave functions (e.g. [4]). Taking these expressions into account, the three parameters $\lambda_{j}$ are plotted as functions of magnetic field in Fig. 2a.
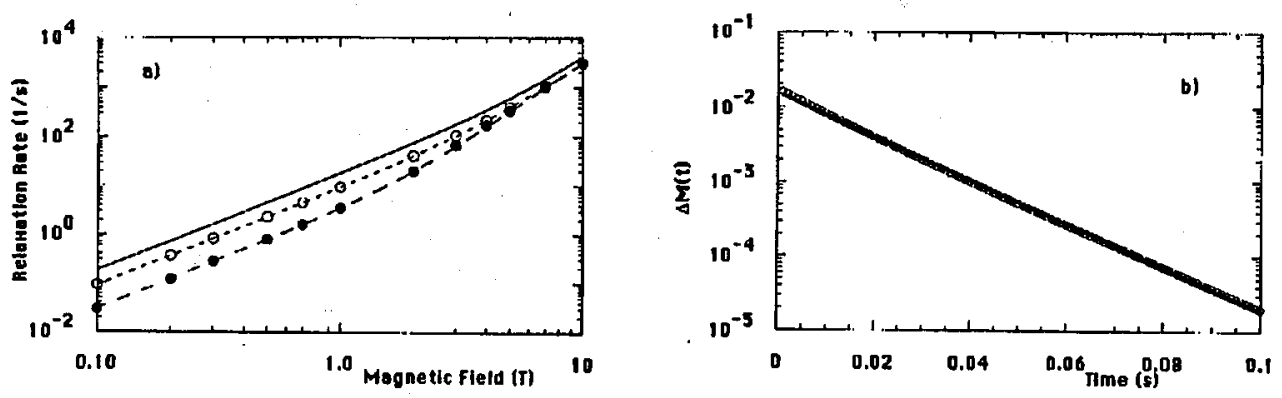

Fig. 2. (a) The fitted relaxation rates (solid points) compared with the calculated ones represented by solid and broken lines (see text). The open circles show $R R 3$ (see text for details). (b) Typical time dependence of magnetization difference $\Delta M=M(t)-M(\infty)$ after a heat pulse at $2 \mathrm{~K}$ and $B=3 \mathrm{~T}$. The solid line represents the exponential function fit.

The results of calculations mentioned above are compared with the results of numerical solutions of differential Eqs. (1) using the fourth-order Runge-Kutta method. The initial conditions were taken as those in heat pulse experiments [2]. It wass assumed that at $t=0$ the lattice temperature is $\Delta T=0.1 \mathrm{~K}$ higher than the spin temperature. The obtained numerical results gave us the temporal behavior of 
magnetization expressed by the sum $M(t)=\sum_{i} n_{i}(t) S_{z_{i}}$. In Fig. $2 \mathrm{~b}$ the difference $\Delta M(t)=M(t)-M(\infty)$ is plotted for $B=3 \mathrm{~T}$ and $T=2 \mathrm{~K}$. As one can see, the decay, after a short transition period, is exponential over many orders of magnitude. The function $F(t)=P \exp (-R t)$ ( $P$ and $R$ are fitting parameters) is fitted to these data and in Fig. 2b we compare the obtained values of $R$ with the relaxation rates $\lambda_{j}$ previously calculated from the rate equations (solid and broken lines). In the same figure we plotted as open circles the values of $R R 3=3\left(W_{\mathrm{u}}+W_{\mathrm{d}}\right)$. They describe relaxation rates between levels 1 and 2 in the two-level approximation or, in our case, in a very high magnetic field approximation. Of course, at a high field only the lowest level is occupied and only the two lowest levels play a role in the relaxation process $(\Delta E / k T \gg 1)$. Therefore, in the experiment $R R 3$ will be observed. As one can see from Fig. 2b, such an approximation $(T=2 \mathrm{~K})$ can be reasonable above $3 \mathrm{~T}$. Of course, this limit is strongly temperature dependent [1].

It is clearly seen that in the system at low field (below $6 \mathrm{~T}$ ) there exist three different relaxation times. Two of them merge above $7 \mathrm{~T}$ and are close (equal at fields above $10 \mathrm{~T}$ ) to $1 / R R 3$. The third one, which is the fastest, goes toward $1 /\left[4\left(W_{\mathrm{u}}+W_{\mathrm{d}}\right)\right]$. The presented calculations show also that with the starting conditions mentioned above (heat pulse) the relaxation could be described by single exponential function during quite a long time interval. In comparison with analytical solutions one can find that the obtained relaxation time is always the longest one in the system, which is due to the starting conditions. One can approximate the system by the two-level model at higher fields, thus the relaxation rate in this field range is given by the sum of probabilities of up (absorption of phonon) and down (emission of phonon) transitions ( $R R 3)$.

\section{Acknowledgments}

The author would like to thank Professor J. Gaj for valuable discussions. The work has been supported by the Committee for Scientific Research through grant \#2 02239101.

\section{References}

[1] A.M. Witowski, Acta Phys. Pol. A 82, 876 (1992); Solid State Commun. 86, 347 (1993).

[2] T. Strutz, A.M. Witowski, P. Wyder, Phys. Rev. Lett. 68, 3912 (1992); T. Strutz, A.M. Witowski, P. Wyder, Rev. Sci. Instrum. 64, 1853 (1993).

[3] J.K. Furdyna, J. Appl. Phys. 64, R29 (1988) and references therein.

[4] K.W.H. Stevens, Rep. Prog. Phys. 30, 189 (1967); K.J. Standley, R.A. Vaughan, Electron Spin Relaxation Phenomena in Solids, A. Hilger, London 1969.

[5] H.A. Weakliem, J. Chem. Phys. 30, 2117 (1962); A.J. Ryskin, A.L. Natadze, S.A. Kazanskii, Zh. Eksp. Teor. Fiz. 64, 910 (1973).

[6] E.R. Andrew, D.P. Tunstall, Proc. Phys. Soc. 78, 1 (1961). 